\title{
Nodal mesenteric marginal zone lymphoma with amyloid deposits, calcification, and ossification
}

\author{
Sotiris Sotiriou ${ }^{1}$ (D) Triantafyllia Koletsa $^{1} \cdot$ Sofia-Eleni Tzorakoleftheraki ${ }^{1} \cdot$ Vassiliki Kotoula $^{1} \cdot$ Stylianos Mantalovas $^{2}$. \\ loannis Kostopoulos ${ }^{1}$
}

Received: 25 October 2018 / Accepted: 6 December 2018 / Published online: 18 December 2018

(C) Springer-Verlag GmbH Germany, part of Springer Nature 2018

\begin{abstract}
Marginal zone lymphomas (MZLs) are rarely associated with amyloid deposits, calcification, and ossification. Herein, we describe a case of a MZL with a primary nodal mesenteric presentation, associated with amyloid deposits, calcification, and ossification, highlighting its clinicopathological features, based on our findings, but also on a brief review of the literature.
\end{abstract}

Keywords Marginal zone lymphoma $\cdot$ Calcification $\cdot$ Ossification $\cdot$ Amyloid deposits

An 81-year-old man has undergone a right colectomy due to a previously diagnosed adenocarcinoma of the hepatic flexure of the colon. On preoperative radiologic evaluation, a mesenteric nodule, measuring $1.9 \mathrm{~cm}$ in greatest diameter with calcification, was observed and was considered to be a metastatic lymph node. Neither splenomegaly nor systemic lymphadenopathy was observed. The patient underwent a right colectomy and the specimen of the right colectomy along with the mesenteric nodule was sent to the pathology department for histologic examination.

Microscopically, the tumor of the hepatic flexure represented a mucinous adenocarcinoma. The mesenteric nodule corresponded to adipose tissue with dense infiltration of neoplastic lymphoid cells, arranged in a solid pattern of growth around a pink amorphous deposition (Fig. 1c). Areas of calcification and ossification were also present (Fig. 1d). The aforementioned cells had intermediate size with pale cytoplasm and regular nuclear membrane with inconspicuous nucleoli (Fig. 1a). On immunohistochemical stains, the neoplastic cells showed the following immunophenotype: CD20+ (Fig. 1b), CD45RA+, BCL2+, CD38-/+, CD3-, CD43-, CD5-, CD23-, CyclinD1-, BCL6-, CD10-, MUM1-,

Sotiris Sotiriou

sotiris_sot@hotmail.com

1 Pathology Department, Faculty of Medicine, Aristotle University of Thessaloniki, 54124 Thessaloniki, Greece

2 3rd Department of Surgery, "AHEPA" University Hospital, Aristotle University of Thessaloniki, Thessaloniki, Greece
DBA44-, TdT-, $\mathrm{k}-$, and $\lambda$-. Ki-67 proliferation index was about $15 \%$. The immunohistochemical stain for CD23 revealed the presence of remnants of follicular dendritic cell meshwork. The examination of Congo red-stained slides under polarized light revealed that the pink amorphous deposits represented amyloid (Insert). Interestingly, amyloid deposits were negative for both $\mathrm{K}$ and $\lambda$ light chains. Although, due to the decalcification process, the quality of DNA extraction products was not satisfying, PCR for immunoglobulin heavy chain (IGH) rearrangement revealed a clonal rearrangement involving the $\mathrm{VC}$ region, confirming the clonal nature of the $\mathrm{B}$ cell population.

The association of amyloid deposits with MZLs is rare [1], although it tends to be more common in cases of primary pulmonary MALT lymphomas [2]. To the best of our knowledge, two case reports of MZL with amyloid deposits associated with calcification and ossification were reported in the English literature. The first case presented as a primary pulmonary MALT lymphoma [3] and the second as a primary orbital MALT lymphoma [4]. Our case represents the third such case, with a primary mesenteric presentation, based on the absence of splenomegaly or lymphadenopathy according to the clinical and radiological findings. On clinical and radiological grounds, there was no evidence of multiple organ amyloid involvement. Our patient did not receive any adjuvant therapy for the lymphoma and there are no signs of relapse 20 months after his initial diagnosis.

The observed nodular pattern of the amyloid deposits in the lung is followed by calcification in about 50\% of the cases [5]. Although amyloid deposits in cases of MZLs tend to be 
Fig. 1 a Medium power view shows neoplastic lymphoid cells with intermediate size, pale cytoplasm, and regular nuclear membrane with inconspicuous nucleoli $(H \& E, \times 400)$. b The neoplastic cells are positive for CD20 (immunoperoxidase with hematoxylin counterstain, $\times 100$ ). c Medium power view of the dense infiltration of neoplastic lymphoid cells, arranged in a solid pattern of growth around a pink amorphous deposition $(\mathrm{H} \& \mathrm{E}, \times 100)$. Insert: The examination of Congo red-stained slide under polarized light shows apple-green birefringence, confirming that the pink amorphous deposits represent amyloid (Congo red-stained slide under polarized light $\times 100$ ). d Areas of calcification and ossification $(\mathrm{H} \& \mathrm{E}, \times 400)$

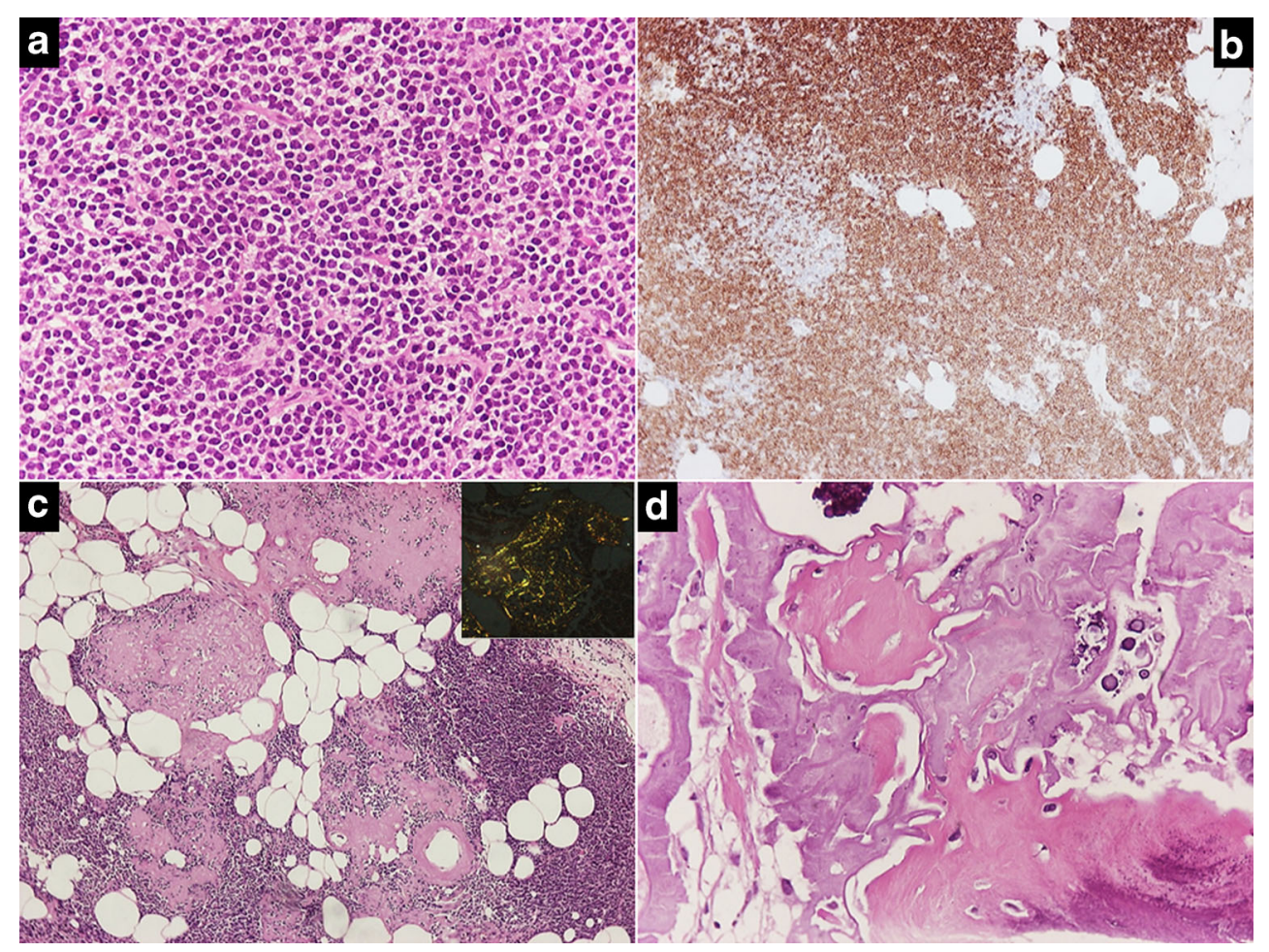

peritumoral and organ confined and do not implicate worse prognosis, rarely multiorgan involvement can be observed [1]. In those cases, the complications can be critical and cause considerable morbidity. Pathologists should be aware of the association between MZLs and amyloid deposition, in order to inform the clinicians for the appropriate follow-up.

\section{Compliance with ethical standards}

Conflict of interest The authors declare that they have no conflict of interest.

\section{References}

1. Zhang Q, Pocrnich C, Kurian A, Hahn AF, Howlett C, Shepherd J, Rizkalla K (2016) Amyloid deposition in extranodal marginal zone lymphoma of mucosa-associated lymphoid tissue: a clinicopathologic study of 5 cases. Pathol Res Pract 212(3):185-189

2. Grogg KL, Aubry M-C, Vrana JA, Theis JD, Dogan A (2013) Nodular pulmonary amyloidosis is characterized by localized immunoglobulin deposition and is frequently associated with an indolent B-cell lymphoproliferative disorder. Am J Surg Pathol 37(3):406412

3. Xiang H, Wu Z, Wang Z, Yao H (2015) Nodular pulmonary amyloidosis and obvious ossification due to primary pulmonary MALT lymphoma with extensive plasmacytic differentiation: report of a rare case and review of the literature. Int J Clin Exp Pathol 8(6):74827487

4. Haas BD, Margo CE (2007) Orbital lymphoma, amyloid, and bone. Ophthalmology 114(6):5-7

5. Urban BA, Fishman EK, Goldman SM, Scott WW Jr, Jones B, Humphrey RL, Hruban RH (1993) CT evaluation of amyloidosis: spectrum of disease. RadioGraphics 13(6):1295-1308 